\title{
Screening und Therapie des frühen Zervixkarzinoms
}

\author{
J.-U. Blohmer \\ Klinik für Frauenheilkunde und Geburtshilfe, Campus Charité Mitte, Medizinische Fakultät der Humboldt-Universität Berlin
}

Das invasive Zervixkarzinom ist als die fünfthäufigste Krebserkrankung weltweit und als zweithäufigste tumorbedingte Todesursache der Frau [1] ein globales Problem, insbesondere in den sogenannten Entwicklungsländern. In den westlichen Industriestaaten zeigt sich seit etwa 20 Jahren neben einer Abnahme der Inzidenz des Zervixkarzinoms um etwa $40 \%$ bei allen Frauen eine ansteigende Prävalenz des Zervixkarzinoms bei jüngeren Frauen. So ist der Anteil der Frauen unter 35 Jahren unter allen Frauen mit einem Zervixkarzinom von 9 auf $25 \%$ in diesem Zeitraum gestiegen [2]. Dieser Trend ist ebenfalls bei der Zunahme der Inzidenz der unmittelbaren Vorstufe der Zervixkarzinome, der hochgradigen zervikalen intraepithelialen Neoplasie (CIN III), insbesondere bei den Frauen, die jünger als 35 Jahre sind, zu beobachten [3].

Mit diesen globalen Problemen des Zervixkarzinoms beschäftigte sich eine Special Session auf dem 35. Annual Meeting der American Society of Clinical Oncology (ASCO) am 16. Mai 1999 in Atlanta, Georgia: «International Symposium on Cervical Cancer: Screening and Treatment of Early Disease, a Global Perspective».

Zunächst wurden von G. Parham (University of Arkansas) verschiedene Studien vorgestellt, die den Wert der Spekuloskopie (Betrachtung der Portio uteri nach Betupfen mit 2-4\%iger Essigsäure bei 4-6facher Vergrößerung mit einer in der Hand gehaltenen Optik bei Tages- oder Raumlicht), der Kolposkopie (binokulare Optik, 10-15fache Vergrößerung, Kaltlichtquelle) und der gynäkologischen Zytologie in der Erkennung und dem Ausschluß der CIN III untersuchten. In einer Studie aus der University Zimbabwe [4] wurden 10.934 Frauen von ausgebildeten Krankenschwestern mit allen drei Methoden untersucht. Die Kolposkopie und Biopsie dienten als Referenzmethoden. Die Sensitivität der Spekuloskopie für die Erkennung der CIN III betrug $77 \%$ (der positive prädiktive Wert allerdings nur 18,6\%), die der Zytologie 44\%, die Spezifität der Zytologie hingegen $91 \%$, und die der Spekuloskopie 64\%. Diese Ergebnisse zeigen einen einfachen Weg für die Früherkennung des Zervixkarzinoms durch die Kolposkopie/Spekuloskopie (hohe Sensitivität) und eine relativ sichere Vermeidung unnötiger Gewebsentnahmen durch die zytologische Untersuchung optisch suspekter Befunde (hohe Spezifität). Dieses Verfahren entspricht allerdings nicht dem in Deutschland üblichen fachärztlichen Zytologie-Screening.
Zur Therapie des frühen (nicht metastasierten) Zervixkarzinoms hat Sardi [5] (Buenos Aires University) seine aktualisierten, noch nicht publizierten Studienergebnisse zur neoadjuvanten Therapie des Zervixkarzinoms vorgestellt. Patientinnen mit einem Plattenepithelkarzinom der Cervix uteri erhielten ohne (!) histologischen oder apparativen Ausschluß einer Lymphknotenmetastasierung (keine Computertomographie, keine Kernspintomographie, keine Lymphographie) nach Randomisation in der Untersuchungsgruppe $(\mathrm{n}=102 \mathrm{~Pa}-$ tientinnen) präoperativ 3 Zyklen mit Cisplatin $50 \mathrm{mg} / \mathrm{m}^{2}$ (über $15 \mathrm{~min}, \mathrm{~d} 1$ ), Vincristin $1 \mathrm{mg} / \mathrm{m}^{2}$ (Bolusinjektion, d1), Bleomycin $25 \mathrm{mg} / \mathrm{m}^{2}$ (Infusion über $6 \mathrm{~h}, \mathrm{~d} 1-3$ ) im Abstand von 10 Tagen. Danach erfolgte die Laparotomie und bei möglicher kompletter (histologischer) Resektion des gesamten Tumors die radikale Hysterektomie nach Wertheim-Meigs mit anschließender adjuvanter pelviner Bestrahlung (eine Extendedfield-Bestrahlung erhielten nur die Patientinnen mit nachgewiesener paraaortaler Lymphknotenmetastasierung). Die Patientinnen der Kontrollgruppe $(\mathrm{n}=103)$ wurden ohne präoperative Chemotherapie laparotomiert und radikal operiert, wenn operationstechnisch möglich, und bestrahlt. Die Patientinnen, die nicht radikal $\mathrm{zu}$ operieren waren, erhielten ausschließlich eine pelvine Bestrahlung. Von den neoadjuvant behandelten Patientinnen mit einem «bulky» Zervixkarzinom (IB2, n=61) konnte tatsächlich bei allen klinisch als operabel eingeschätzten Patientinnen das Karzinom histologisch komplett reseziert (R0-Resektion) werden, bei den Patientinnen der Kontrollgruppe mit einem IB2-Karzinom $(n=56)$ nur bei $85 \%(n=48)$. Bei den operierten, neoadjuvant behandelten Patientinnen im Stadium IIB waren die histologischen Prognosefaktoren signifikant besser als bei den Patientinnen der Kontrollgruppe: $\mathrm{pN} 1: 25$ versus $41 \%(\mathrm{p}=0,05), \mathrm{V} 1: 28$ versus $59 \%(\mathrm{p}=0,0001)$, parametraner Befall: 34 versus $76 \%$ $(p=0,0001)$. Die bessere Operabilität war eventuell eine Ursache für das verbesserte Gesamtüberleben und längere erkrankungsfreie Überleben der chemotherapeutisch behandelten Gruppe der «bulky» (Tumor größer als $4 \mathrm{~cm}$ ) Zervixkarzinome gegenüber der nichtbehandelten Kontrollgruppe innerhalb einer medianen Beobachtungszeit von inzwischen 94 Monaten. Bei den Zervixkarzinomen mit einer Größe zwischen 2 und $4 \mathrm{~cm}$ konnten Sardi et al. [5] keinen signifikanten Unterschied zwischen beiden Therapien feststellen. Sardi

\begin{tabular}{ll}
\hline KARGER & @ 1999 S. Karger GmbH, Freiburg \\
Fax +49 761 4520714 & Accessible online at: \\
$\begin{array}{l}\text { E-mail kargergmbh@aol.com } \\
\text { www.karger.com }\end{array}$ & http://BioMedNet.com/karger
\end{tabular}

Jens-Uwe Blohmer

Medizinische Fakultät der Humboldt-Universität Berlin,

Klinik für Frauenheilkunde und Geburtshilfe, Campus Charité Mitte

Schumannstraße 20/21, D-10117 Berlin (Deutschland)

Tel. +49 30 2802-5733, Fax -2802-4446

E-mail jens-u.blohmer@charite.de 
hat seine Ergebnisse mit denen der jüngst publizierten Studien zur simultanen Radio-Chemotherapie von Keys et al. [6] verglichen. In dieser Studie wurden 369 Frauen mit einem «bulky» Zervixkarzinom im Stadium IB (T1b2) und nach Ausschluß (bildgebend oder histologisch) einer Lymphknotenmetastasierung (pN0 und N0) nach Randomisierung entweder ausschließlich bestrahlt (perkutan und intrakavitär mit 75 Gy im Punkt A und 55 Gy im Punkt B) oder simultan mit einer Chemotherapie (Cisplatin $40 \mathrm{mg} / \mathrm{m}^{2}$ wöchentlich, 6 Zyklen) und gleicher Strahlentherapie behandelt und 3-6 Wochen später adjuvant hysterektomiert.

Ein Unterschied der Studie von Sardi et al. [5] zu den anderen randomisierten Studien [7] zur neoadjuvanten Chemotherapie des Zervixkarzinoms ist die kurze Therapiedauer und die relativ hohe Dosisdichte. Obgleich beide Studien [5 und 6] hinsichtlich ihrer Patientinnenselektion (die Patientinnengruppe von Sardi et al. ist aufgrund des fehlenden Ausschlusses von pN1 prognostisch schlechter), der Nachbeobachtungszeit und des Studiendesigns verschieden sind, gleichen sie sich in einer Aussage: Die Patientinnen mit einer Chemotherapie haben signifikant länger überlebt ( 80 und $83 \%$ vs. 61 und $74 \%$ ) und länger erkrankungsfrei gelebt (79 und $79 \%$ vs. 59 und 63\%). Diese Aussage trifft auch auf die anderen zusammen mit der Arbeit von Keys et al. [6] publizierten Studien [8 und 9] zur simultanen Radio-Chemotherapie im Vergleich zur alleinigen Strahlentherapie beim Zervixkarzinom zu [10]. Publizierte
Daten zu ausreichend großen, randomisierten Studien zur adjuvanten Chemotherapie beim nach Wertheim-Meigs operierten Zervixkarzinom fehlen noch. In Deutschland wird zur Zeit eine randomisierte Studie mit einer adjuvanten sequentiellen Chemo-(4 Zyklen Ifosfamid 1,6 g/m², d1-3, und Carboplatin AUC4 d1, q 21d)-Radiotherapie durchgeführt (Anfragen an den Verfasser). Die Ergebnisse der Phase-IIStudie [11] waren mit $85 \%$ rezidivfreien Patientinnen in einer medianen Nachbeobachtungszeit von 30 Monaten bei einer relativ hohen, aber behandelbaren hämatologischen und nichthämatologischen Toxizität vielversprechend. Wegen der hohen Anämierate (30\% der Zyklen Grad 1-2 und 10\% Grad 3-4) der Patientinnen der Phase-II-Studie bei dem prognostisch ungünstigen Einfluß einer Anämie auf bestrahlte Patientinnen mit einem Zervixkarzinom [12] werden die Patientinnen dieser Studie in einen Therapiearm mit obligatorischer Erythropoetingabe randomisiert, um den Einfluß der zusätzlichen Gabe des Erythropoetins auf das erkrankunsfreie Intervall zu untersuchen.

Bis die vielversprechende therapeutische und prophylaktische Vakzinierung gegen die high-risk Humanen Papillomaviren in die klinische Praxis eingeführt ist, liegt die Lösung des globalen Problems Zervixkarzinom in einem effektiven Screening nach präinvasiven Zervixkarzinomen (CIN III), in einer aufgeklärten Bevölkerung und in einer wirksamen, eine Chemotherapie enthaltenden Therapie der invasiven Stadien.

\section{Literatur}

1 Parkin DM, Laara E, Muir CS: Estimates of the worldwide frequency of sixteen major cancer in 1980. Int J Cancer 1988;41:184-197.

2 Luschin-Ebengreuth G: Cervical cancer. Prognostic factors. Age; in Burghardt E, Webb MJ, Monaghan JM, Kindermann G (eds): Surgical Gynecologic Oncology. Stuttgart, Thieme, 1993, pp 322-324.

3 Blohmer JU, Schmalisch G, Klette I, Grineisen Y, Kohls A, Guski H, Lichtenegger W: Increase in incidence of intraepithelial neoplasms of the cervix uteri (CIN) in young women in Mitte district of Berlin, Germany. Acta Cytol 1999;43:195-200.

4 Kitchener HC, Symonds P: Detection of cervical intraepithelial neoplasia in developing countries. Lancet 1999;353:856-857.

5 Sardi J, Giaroli A, Sananes C, Ferreira M, Soderini A. Bermudez A, Snaidas L, Vighi S, Gomez Rueda N, di Paola G: Long-term follow up of the first randomized trial using neoadjuvant chemotherapy in stage $\mathrm{Ib}$ squamous carcinoma of the cervix: The final results. Gynecol Oncol 1997;67:61-69.
6 Keys HM, Bundy BN, Stehman FB, Mudersbach LI, Chafe WE, Suggs III CL, Walker JL, Gersell D: Cisplatin, radiation, and adjuvant hysterectomy compared with radiation and adjuvant hysterectomy for bulky stage Ib cervical carcinoma. N Engl J Med 1999;340:1154-1161.

7 Vermorken JB: Neoadjuvant chemotherapy (NACT) in cervical cancer: Still promising? Ann Oncol 1992 3:97-101.

8 Morris M, Eifel PJ, Lu J, Grigsby PW, Levenback C, Stevens RE, Rotman M, Gershenson DM, Mutch DG: Pelvic radiation with concurrent chemotherapy compared with pelvic and para-aortic radiation for high-risk cervical cancer. N Engl J Med 1999;340: 1137-1143.
9 Rose PG, Bundy BN, Watkins EB, Thipgen JT, Deppe G, Maiman MA, Clarke-Pearson DL, Insalaco S: Concurrent cisplatin-based radiotherapy and chemotherapy for locally advanced cervical cancer. N Engl J Med 1999;340:1144-1153.

$10 \mathrm{McNeil} \mathrm{C}$ : New standard of care for cervical cancer sets stage for next questions. J Natl Cancer Inst 1999;91:500-501.

11 Blohmer JU, Paepke S, Kissner L, Sehouli J, Elling D, Lichtenegger W: Combined adjuvant radiochemotherapy in patients with cervical carcinoma and a high risk of recurrence. Acta Obstet Gynecol Jap 1999;51(suppl):S618(IS2).

12 Girinski T, Pejovic Lenfant MH, Bourhis J, Campana F, Cosset JM, Petit C, Malaise EP, Haie C, Gerbaulet A, Chassagne D: Prognostic value of hemoglobin concentrations and blood transfusions in advanced carcinoma of the cervix treated by radiation therapy: Results of a retrospective study of 386 patients. Int J Radiat Oncol Biol Phys 1989;16:37-42. 